\title{
Endurance Testing in A Group of Indian Athletes and Non Athletes
}

\author{
Amrith Pakkala ${ }^{1}$, Ashwini K Shetty ${ }^{2 *}$ \\ ${ }^{1}$ Dept of Physiology, Shridevi Institute of Medical Sciences \& Research Hospital, Tumakuru, Karnataka, India \\ ${ }^{2}$ Dept of Physiology, MVJ Medical College\& Research Hospital, Hoskote, Karnataka, India
}

\begin{abstract}
Background: The role of estrogen on the heart is well known in the normal course of the menstrual cycle. Significant increase in both progesterone (37\%) and estradiol (13.5\%), whereas no change in plasma FSH \& LH was observed in exercising women in previous studies. Therefore this study was intended to see the limitations of the pulmonary system in adaptability to exercise in proliferative phase of menstrual cycle in perimenopausal women.
\end{abstract}

Material and Methods: Healthy adult females between 42-45 years who regularly undergo training and participate in competitive middle distance running events for at least past 3 years were considered in the athlete group whereas the non-athlete group did not have any such regular exercise program. Dynamic heart functions were measured in both groups before exercise was evaluated following standard procedure of treadmill testing.

Results: It was observed that exercise per se cause a statistically significant change in dynamic heart function parameters in either of the groups.

Summary and Conclusion: This finding supports the hypothesis that the cardiovascular system is normally the most limiting factor in the delivery of oxygen even under the predominant influence of estrogen in proliferative phase which is further accentuated by exercise.

Keywords: Proliferative Phase, Estrogen in Exercise, Dynamic Heart Functions, Adaptability

\section{Introduction}

The role of hormones on the healthy cardiovascular system in delivering oxygen to meet the demands of various degrees of exercise has been a matter of differences in opinion. There are conflicting reports that the cardiovascular System is normally the most limiting factor in the delivery of oxygen to the muscles during maximal muscle aerobic metabolism whereas others do not subscribe to this ${ }^{1}$. Within this context it is appropriate to study the effect of proliferative phase of menstrual cycle on heart functions after exercise.

Mechanical constraints on exercise hyperpnoea have been studied as a factor limiting performance in endurance athletes' ${ }^{\left[{ }^{2}\right]}$. Others have considered the absence of structural adaptability to physical training as one of the "weaknesses" inherent in the healthy cardiovascular system response to exercise ${ }^{[3]}$

Heart functions are an important part of functional diagnostics ${ }^{[4]}$, aiding selection and optimization of training and early diagnosis of sports pathology. Assessment of exercise response of dynamic lung functions in the healthy cardiovascular system in the trained and the untrained has a role in clearing gaps in the above areas especially a special group like perimenopausal women.

\section{Material and Methods}

The present study was conducted as a part of cardiopulmonary efficiency studies on two groups of non-athletes $(\mathrm{n}=10)$ and athletes $(\mathrm{n}=10)$ comparable in age $\&$ sex.

Informed consent was obtained and clinical examination to rule out any underlying disease was done. Healthy adult females between $42-45$ years who regularly undergo training and participate in competitive middle distance running events for at least past 3 years were considered in the athlete group whereas the non-athlete group did not have any such regular exercise program. Smoking, clinical evidence of anemia, obesity, involvement of cardiorespiratory system was considered as exclusion criteria. Menstrual history was ascertained to confirm proliferative phase of menstrual cycle.Detailed procedure of exercise treadmill test and computerized spirometry was explained to the subjects.

Dynamic heart functions were measured in both groups before exercise was evaluated following standard procedure of treadmill testing. All subjects were made to undergo maximal exercise testing to $\mathrm{VO} 2$ max levels on a motorized treadmill. 
After exercise, the assessment of dynamic lung functions was repeated. All these set of recordings were done on both the non-athlete as well as the athlete groups.

Statistical analysis was done using paired students t-test for comparing parameters within the group before \& after exercise testing and unpaired t-test for comparing the two groups of subjects.

A p-value of $<0.01$ was considered as significant.

\section{Results}

It is clear from table 1 that the two study groups are anthropometrically similar and comparable.The dynamic cardiopulmonary functions did not show any significant changes in the non athlete and athlete groups as shown in table $2 \& 3$.It was observed that exercise per se cause a statistically significant change in dynamic heart function parameters in either of the groups.

\section{Discussion}

Considerable information can be obtained by studying the exercise response of dynamic lung functions in untrained and trained subjects.

Intra group comparison is helpful in noting the exercise response and inter-group comparison in evaluating adaptations of the respiratory system to training.

On comparing the anthropometric data of the two study groups it is clear that the age \& sex matched subjects have no statistically significant difference in height taking a pvalue of $<0.05$ as significant.

VO2 max values were higher in controls and was statistically significant $(\mathrm{P}<0.001)$. This observation is expected in view of the training stimulus and adaptability of both the pulmonary system and the cardio vascular system. VO2 max is an objective index of the functional capacity of the body's ability to generate power.

Forced vital capacity (FVC) is the volume expired with the greatest force and speed from TLC and FEV1 that expired in the $1^{\text {st }}$ second during the same maneuver. The FEV1 was initially used as an indirect method of estimating its predecessor as the principal pulmonary function test, the maximal breathing capacity ${ }^{[8]}$. On comparing the response of exercise within the two study groups and in between them, there is no statistically significant difference in FVC \& FEV1 under any condition. A normal FEV1/FVC ratio is observed always.

Table No. 1: Comparison of anthropometric data \& V02 max of non-athletes \& athletes with statistical analysis.

\begin{tabular}{|c|c|c|c|c|}
\hline Parameter & Non-Athletes & Athletes & P- value & Remarks \\
\hline Age $(\mathrm{Yr})$ & $43.51 \pm 2.62$ & $43.48 \pm 2.84$ & $<0.10$ & Not significant \\
\hline Height $(\mathrm{cm})$ & $160.71 \pm 7.51$ & $155.91 \pm 7.25$ & $<0.10$ & Not significant \\
\hline Weight $(\mathrm{kg})$ & $52.65 \pm 5.66$ & $55.44 \pm 6.26$ & $<0.05$ & Not significant \\
\hline BMl $\left(\mathrm{kg} / \mathrm{m}^{2}\right]$ & $22.01 \pm 2.47$ & $21.61 \pm 1.75$ & $<0.10$ & Not significant \\
\hline VO2 $\max (\mathrm{lit} / \mathrm{min})$ & $2.49 \pm 0.15$ & $2.96 \pm 0.28$ & $<0.001$ & Highly Significant \\
\hline
\end{tabular}

Table No. 2: Comparison of Dynamic cardiopulmonary Functions of Non- Athletes before exercise testing (BE) \& after exercise testing (AE) with statistical analysis. NON-ATHLETES $(n=10)$.

\begin{tabular}{|c|c|c|c|c|}
\hline Parameter & BE & AE & P-value & Remarks \\
\hline Heart Rate bpm & 76.09 & 86.00 & $<0.10$ & Not Significant \\
\hline FEV1 (L) & $3.10 \pm 0.51$ & $2.98 \pm 0.05$ & $<0.05$ & Not Significant \\
\hline FEV1/FVC & 0.951 & 0.962 & $<0.05$ & Not Significant \\
\hline
\end{tabular}

$P<0.01$ is considered significant

Table No. 3; Comparison of Dynamic cardiopulmonary functions of Athletes before exercise testing (BE) \& after exercise testing (AE) with statistical analysis. ATHLETES $(n=10)$.

\begin{tabular}{|c|c|c|c|c|}
\hline Parameter & BE & AE & P-value & Remarks \\
\hline Heart Rate bpm & 70.10 & 78.08 & $<0.10$ & Not Significant \\
\hline FEV1 (L) & $3.11 \pm 0.51$ & $2.99 \pm 0.04$ & $<0.05$ & Not Significant \\
\hline FEV1/FVC & 0.95 & 0.97 & $<0.05$ & Not Significant \\
\hline & & & & \\
\hline
\end{tabular}

$P<0.01$ is considered significant 
Another way of looking at forced expiration is to measure both expiratory flow and the volume expired. The maximum flow obtained can be measured from a flow -volume curve is the peak expiratory flow rate (PEFR). The peak flow occurs at high lung volumes and is effort dependent. Flow at lower lung volumes is effort independent. Flow at lower lung volumes depends on the elastic recoil pressure of the lungs and the resistance of the airways upstream or distal to the point at which dynamic compression occurs. Measurements of flow at low lung volumes, mid expiratory flow [MEF $25 \%$ to $75 \%$ ] are often used as indices of peripheral or small airways resistance ${ }^{[8]}$

On examining Table $2 \&$ Table 3 it is clear that exercise per se does not cause a statistically significant change in dynamic lung function parameters MMEF, PEFR, MEF $25 \%$ to $75 \%$ in either of the groups. This finding supports the hypothesis that the respiratory system is not normally the most limiting factor in the delivery of oxygen. These findings are in line with other studies Bonen A et al and Jurkowski JE et al ${ }^{[10,11]}$

Thirty minutes of exercise at $74 \%$ of $\mathrm{VO} 2$ was found to cause a significant increase in both progesterone $(37 \%)$ and estradiol (13.5\%), whereas no change in plasma FSH \& LH was observed in exercising women ${ }^{[7]}$; others have confirmed these findings ${ }^{[8]}$. This finding supports the hypothesis that the respiratory system is not normally the most limiting factor in the delivery of oxygen even under the predominant influence of a sedentary and obese life style of the group studied.

\section{References}

1. Cunningham GR, Tindall DJ, Means AR. Differences in steroid specificity for rat ABP. Steroids 1979;33:261-276

2. Amrith Pakkala. Mid- career blues in healthcare workers: A physiological approach in ethical management. J Mid-life Health 2010;1/1: 35-37

3. Frye CA. Steroids, reproductive endocrine function and affect- A review. Minerva Ginecol 2009; 61: 541-562

4. Guyton AC, Hall JE, editors -Text Book of Medical Physiology ,11th ed .Saunders, 2006 :1061-62.

5. Johnson BD, Saupe KW, Dempsey JA -Mechanical constraints on exercise hypernea in endurance athletes. $\mathrm{J}$ Appl Physiol 1992 Sep; 73(3): 874- 86

6. Dempsey JA, Johnson BD, Saupe KW-Adaptations and limitations in the pulmonary system during exercise. Chest 1990 Mar; 97(3 Suppl):81s -87s

7. Andziulis A, Gocentas A, Jascaniniene N, Jaszczanin J, Juozulynas A, Radzijewska M -Respiratory function dynamics in individuals with increased motor activity during standard exercise testing. Fiziol ZH 2005; 51(4):80-95.

8. Seaton A, Seaton D, Leitch AG, editors -Crofton and Douglas's Respiratory Diseases, 5th ed. Oxford: Oxford University press, 2000:43-45

9. Ganong WF, Review of Medical Physiology, 22nd ed $2005 ; 444$

10. Bonen A, Ling WY, MacIntyre KP, et al. Effects of exercise on the serum concentrations of FSH, LH, progesterone and estradiol. Eur J Appl Physiol 1979;43:15

11. Jurkowski JE, Jones NL, Walker C, et al. Ovarian hormonal responses to exercise. Eur J Appl Physiol 1978; 44:109

*Corresponding author:

Dr Ashwini K Shetty MD Associate Professor, Dept of Physiology, MVJ Medical College\& Research Hospital, Hoskote, Karnataka, India. Email: pan1832@gmail.com

Financial or other Competing Interests: None. 